\title{
The decay of a dipolar vortex in a weakly dispersive environment
}

\author{
Edward R. Johnson \& Matthew N. Crowe \\ Department of Mathematics, University College London, London, WC1E 6BT, UK
}

(Received xx; revised xx; accepted xx)

A simple model is presented for the evolution of a dipolar vortex propagating horizontally in a vertical slice model of a weakly stratified inviscid atmosphere, following the model of Flierl \& Haines (Phys Fluids 6, 3487-3497, 1994) for a modon on the $\beta$-plane. The dipole is assumed to evolve to remain within the family of Lamb-Chaplygin dipoles but with varying radius and speed. The dipole loses energy and impulse through internal wave radiation. It is argued, and verified against numerical solutions of the full equations, that an appropriately defined centre vorticity for the dipole is closely conserved throughout the flow evolution. Combining conservation of centre vorticity with the requirement that the dipole energy loss balances the work done on the fluid by internal wave radiation gives a model that captures much of the observed dipole decay. Similar results are noted for a cylindrical dipole propagating along the axis of a rotating fluid when the dipole axis is perpendicular to the axis of rotation and for a spherical vortex propagating horizontally in a weakly stratified fluid. The model extends to fluids of small viscosity and so provides an estimate for the relative importance of wave drag and dissipation in dipole decay.

\section{Introduction}

Compact vortices can be remarkably long-lived features of many fluid flows. Nycander \& Isichenko (1990) observe that although monopolar vortices tend to be more commonly observed, vortex dipoles (consisting of two strongly bound, oppositely signed vortices), due to their ability to self-propagate over large distances, could be just as important for transport. Velasco Fuentes \& van Heijst (1994) note that dipoles occur in stratified fluids (Flór \& van Heijst 1994), in rotating fluids (Flierl et al. 1983), in the wake of a cylinder moving through a soap film (Couder \& Basdevant 1986) and in magnetohydrodynamic flows (Nguyen Duc \& Sommeria 1988). Questions then arise as to how long lived the dipoles are, and through what mechanism they decay. In many experiments viscous effects are the dominant dissipative effect: Flór \& van Heijst (1994) and Flór et al. (1995) discuss the viscous decay of dipoles in a strongly stratified fluid and Nielsen \& Rasmussen (1997) present numerical integrations showing viscous decay in a homogeneous fluid. Nycander \& Isichenko (1990) consider the general problem of dipoles moving in a weakly inhomogeneous medium and present two mechanisms for dipole decay through the loss of enstrophy. First, a vortex moving in the direction of the gradient of the inhomogeneity (north-south on a rotating sphere or vertically in a stably stratified fluid) generates "ghost vortices" (McWilliams \& Zabusky 1982) and hence loses enstrophy to conserve total enstrophy. Second, when a dipole oscillates along the background gradient its size changes and enstrophy is lost through the separatrix between the dipole and the ambient fluid. Brion et al. (2014) show that an instability also leads to fluid being shed from the rear of a dipole. Shedding is shown to be important in the analysis below.

This paper aims to demonstrate briefly another mechanism for dipole decay present when a dipole propagates in a weakly dispersive medium that extracts energy from 


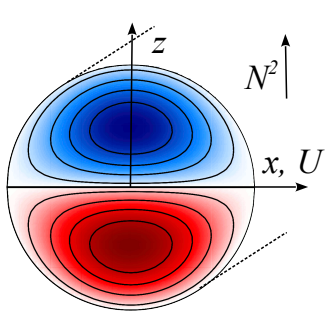

(a)

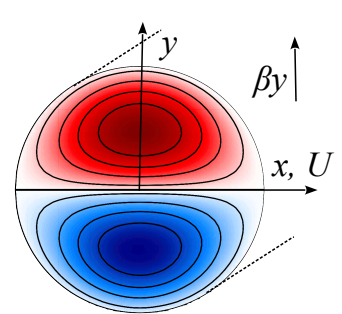

(b)

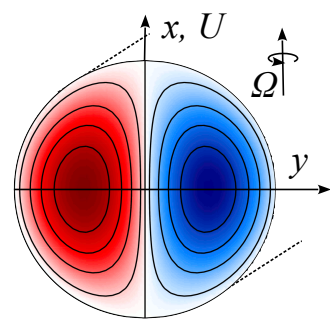

(c)

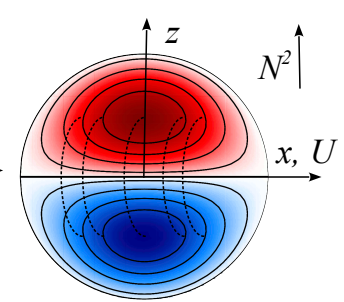

(d)

Figure 1: A schematic showing the cross-sections of the dipole configurations considered. below. In each case the dipole cross-section has radius $a$ and the vortex is advancing along the $x$-axis at speed $U$. (a) A cylindrical vortex in a vertical slice stratified flow model with $O z$ vertical. The cross-section lies in the $O x z$ plane and the flow is invariant in the $O y$ direction $(\S 2)$. (b) A $\beta$-plane modon in the horizontal $O x y$ plane invariant in the $O z$ direction with $O y$ northward (§3). (c) A cylindrical vortex in a frame rotating at angular velocity $\Omega$ about the $x$-axis. The cross-section lies in the $O x y$ plane and the flow is invariant in the $O z$ direction $(\S 5.1)$. (d) A spherical vortex in a stratified flow with $O z$ vertical $(\$ 5.2)$.

the vortex through the work done by the wave drag. Section 2 describes the motion in a two-dimensional vertical-slice weakly stably stratified model. Nielsen \& Rasmussen (1997) show that the Lamb-Chaplygin vortex dipole (Meleshko \& van Heijst 1994) (LCdipole) arises naturally in a flow initialised by a jet and so the the problem is posed in $\S 2$ in terms of a modified LC-dipole. Section 3 shows that the analysis translates directly to the problem of a modon (Larichev \& Reznik 1976; Flierl 1987) propagating westwards under the $\beta$-plane quasigeostrophic approximation for a barotropic ocean, reproducing results obtained using an adjoint method in Flierl \& Haines (1994) (FH) - in particular the algebraic decay of the radius and speed of the modon. Numerical solutions are presented that confirm the numerical integrations in $\mathrm{FH}$ and the close conservation of an appropriately defined centre vorticity for the modon. Section 4 returns to discussion of the slow evolution of the LC-dipole in stratified flow using the requirement of conservation of centre vorticity to derive the decay rate of the dipole radius and speed and the numerical model of $\S 3$ to test the model assumptions and predictions.

Section 5 notes, following Bretherton (1967), that the stratified results translate immediately to the problem of an LC-dipole propagating parallel to the axis of rotation of a weakly rotating homogeneous fluid and gives equivalent results for the decay of a Hill's (Hill 1894) vortex propagating horizontally in a weakly stratified flow. Figure 1 gives a schematic of the cases considered. Positive vorticity is shown in red and negative vorticity blue. The sign of the dipole is chosen so that the dipole propagates in the positive $x$-direction. This means that the dipole sign reverses between parts (a) and (b) as the vortex $(y)$ axis in (a) points into the page whereas the vortex $(z)$ axis in (b) points out of the page. Horizontally propagating dipolar vortices in a vertical slice model similar to (a) have been observed experimentally as mode-two solitons at the diffuse interface of two-layer stratified flows. Kamachi \& Honji (1982) show closed streamline patterns in the co-moving frame and Salloum et al. (2012) and Carr et al. (2015) present vorticity plots clearly showing the dipolar structure. In these experiments, however, the fluid is homogeneous away from the interface and so no energy is radiated away from the interface. Provided it travels faster than the linear mode-two wave, the dipole also radiates no energy along the interface and any decay is due to viscous effects. 
Section 6 extends the model to include viscous dissipation, relating the results to the homogeneous-fluid results of van de Fliert (1996) and Nielsen \& Rasmussen (1997), and $\S 7$ discusses the results briefly.

\section{Two-dimensional stratified flow}

Consider a uniformly stratified inviscid, diffusionless fluid which in its undisturbed state has constant buoyancy frequency $N$. Introduce Cartesian axes $O x^{\prime} y z$ fixed in the fluid with $O z$ vertical and take the motion to be two-dimensional in the sense that the flow is independent of the coordinate $y$, being the same in all vertical slices $y=$ constant with all quantities treated below as two-dimensional but corresponding to three-dimensional quantities taken per unit width in the $y$-direction. Let the fluid be disturbed by a circular LC-dipole of radius $a$ propagating in the $O x^{\prime}$ direction at speed $U$, such that the inverse Froude number, the ratio of the vortex scale to the scale of internal waves that can stand behind the propagating vortex, $\epsilon_{N}=N a / U$, is small (figure 1a). Now consider the leading order flow in the limit $\epsilon_{N} \rightarrow 0$ so that on the scale of the vortex the leading order flow is nonlinear but unaffected by stratification. On the scale of the internal wave wake the vortex appears as a forcing region of area $\epsilon_{N}^{2}$ and so is governed by linear dynamics. The internal wave pressure field acts over a length of order $\epsilon_{N}$ to exert a drag of order $\epsilon_{N}^{3}$ on the vortex. This weak drag causes the vortex to be modified over a timescale $\epsilon_{N}^{3} a / U$, long compared to the advection time $a / U$. As a modelling hypothesis it will be supposed that the response of the vortex to this drag is to evolve so as to remain an LC-dipole but with radius and speed varying slowly in time. The internal wave wake is set up in times of order $a / U$ and so can be taken as quasi-steady during the vortex evolution. The solution requires three components described briefly below: the dipole energy $(\$ 2.2)$, the wave drag (§2.3) and an appropriate vortex centre $(\S 2.4)$.

\subsection{The governing equations}

Let the velocity relative to the fixed axes $O x^{\prime} y z$ be $\boldsymbol{u}=u \hat{\boldsymbol{x}}^{\prime}+w \hat{\boldsymbol{z}}$ and buoyancy acceleration be $b$. For two-dimensional flows in an otherwise static, stably stratified flow of buoyancy frequency $N$, the equations of motion can be written in coordinates moving at the vortex speed $U$ as

$$
\begin{aligned}
\left(\partial_{t}-U \partial_{x}\right) u+u u_{x}+w u_{z} & =-(1 / \rho) p_{x}, \\
\left(\partial_{t}-U \partial_{x}\right) w+u w_{x}+w w_{z}-b & =-(1 / \rho) p_{z}, \\
\left(\partial_{t}-U \partial_{x}\right) b+u b_{x}+w b_{z}+N^{2} w & =0 \\
u_{x}+w_{z} & =0 .
\end{aligned}
$$

where $\rho$ is a constant reference density and $x=x^{\prime}-\int^{t} U\left(t^{\prime}\right) \mathrm{d} t^{\prime}$. This system gives an energy conservation equation in the form

$$
\left(\partial_{t}-U \partial_{x}\right) E_{b}+\nabla \cdot\left(E_{b} \boldsymbol{u}+p \boldsymbol{u}\right)=0, \quad E_{b}=\frac{1}{2} \rho\left(u^{2}+w^{2}\right)+\rho b^{2} / N^{2} .
$$

Introducing the streamfunction $\psi$, defined by $(u, w)=\left(\psi_{z},-\psi_{x}\right)$, and eliminating the pressure then gives for steady flow,

$$
\begin{aligned}
\partial(-U z+\psi, \eta)-b_{x} & =0, \\
\partial\left(-U z+\psi, b+N^{2} z\right) & =0,
\end{aligned}
$$

where $\eta=\nabla^{2} \psi$ is the $y$-component of vorticity and $\partial(.,$.$) denotes the Jacobian. Simple$ nonlinear solutions of (2.3) follow by looking for Long's model solutions (Long 1955) 
where the total density $b+N^{2} z$ is a linear function of the total streamfunction $-U z+\psi$, i.e. $b+N^{2} z=\alpha(-U z+\psi)$, for some $\alpha$. For streamlines originating upstream, where $b$ and $\psi$ vanish, this form requires that $\alpha=-N^{2} / U$ but within the vortex $\alpha$ is arbitrary. Then $(2.3 a)$ can be written

$$
\partial(-U z+\psi, \eta-\alpha z)=0
$$

A simple nonlinear solution is again given by choosing $\eta-\alpha z$ to be a linear function of $-U z+\psi$, i.e. $\eta-\alpha z=\gamma(-U z+\psi)$, for some $\gamma$. For streamlines originating upstream this form requires that $\alpha=U \gamma$ but within the vortex $\gamma$ is arbitrary. The governing equation outside the vortex thus becomes

$$
\begin{aligned}
\nabla^{2} \psi+\kappa^{2} \psi & =0 \\
b & =-N^{2} \psi / U,
\end{aligned}
$$

where $\kappa=N / U$ is the wavenumber of the standing internal wave wake. Multiplying (2.5a) by $\psi$ and integrating over the region exterior to the vortex shows that the energy in the internal wave wake is equipartitioned between kinetic and potential energy as expected,

$$
\int\left(u^{2}+w^{2}\right)=\int\left(\psi_{x}^{2}+\psi_{z}^{2}\right)=\kappa^{2} \int \psi^{2}=\int b^{2} / N^{2} .
$$

Now suppose that relationship $(2.5 b)$ between the density and streamfunction continues to hold within the vortex (so in the absence of stratification the perturbation density vanishes). Then the governing equation inside the vortex can be written

$$
\nabla^{2} \psi+K^{2} \psi=U\left(K^{2}-\kappa^{2}\right) z, \quad b=-N^{2} \psi / U,
$$

where $K$ is the internal wavenumber of the vortex. For $K \neq \kappa$ the energy of the vortex does not satisfy (2.6) and is not equipartitioned between kinetic and potential energy. Larichev \& Reznik (1976) and Flierl (1987) derive system (2.5), (2.7) with $\kappa^{2}$ replaced by $-(\beta / U)$ as the governing equation for eastward propagating dipolar vortices, modons, in a barotropic rigid-lid ocean under the $\beta$-plane approximation and give solutions for circular dipoles surrounded by an exponentially decaying disturbance field. This form of solution requires the modon speed $U$ to be positive so as to lie outside the range of Rossby wave phase speeds. Westward propagating modons, $U<0$, have a speed that coincides with a Rossby wave phase speed. This permits standing Rossby lee-wave wakes that remove momentum and energy from the modon and $\mathrm{FH}$ quantify the subsequent modon decay. As there is no preferred horizontal direction for internal waves the dipoles in the stratified flow here decay irrespective of their direction of propagation.

\subsection{The Lamb-Chaplygin dipole}

In the absence of background stratification $(\kappa=0)$ system $(2.1)$ reduces to the Euler equations. Equations (2.5), (2.7) have the LC-dipole solution (Meleshko \& van Heijst 1994),

$$
\psi= \begin{cases}U z+C \mathrm{~J}_{1}(K r) z / r, & r<a \\ U a^{2} z / r^{2}, & r>a,\end{cases}
$$

where $r=\left(x^{2}+z^{2}\right)^{1 / 2}$, and the dipole boundary is the circle $r=a$. The smallest wavenumber $K$ satisfying the no-normal-flow condition, $-U z+\psi=0$ on $r=a$, i.e. $\mathrm{J}_{1}(K a)=0$, is given by $K a=\gamma$ where $\gamma$ is the first positive root of $\mathrm{J}_{1}$ so $\gamma \approx 3.8317$. Once $a$ is determined $K$ is known. Continuity of tangential velocity at the dipole boundary 
gives $C=-2 U / K \mathrm{~J}_{0}(K a)$. The flow outside the dipole can be written

$$
\psi=U a^{2} \frac{\partial}{\partial x}[\arctan (x / z)], \quad r>a .
$$

Along the line $z=0^{+},(2.9)$ gives

$$
\psi=\frac{\pi}{2} U a^{2} \frac{\partial}{\partial x} \operatorname{sgn} x=\pi U a^{2} \delta(x), \quad z=0^{+} .
$$

showing that the velocity field in $r>a$ is precisely that of an irrotational source doublet of strength $2 \pi \rho U a^{2}$ at the origin directed in the positive- $x$ direction.

The impulse of the dipole follows as in (A 2) as

$$
\mu=-\rho \int z \eta=2 \pi \rho U a^{2}
$$

where the integral is taken over the compact support of $\eta$ (here the disc $r<a$ ), and matches the doublet strength. The energy associated with the dipole is simply the kinetic energy

$$
E=-\frac{1}{2} \rho \int \psi \eta=2 \pi \rho a^{2} U^{2}=U \mu,
$$

again noted in the appendix. Under the hypothesis that the dipole remains within the LC-dipole family as it evolves, (2.2) shows that the dipole potential energy remains a small perturbation to the total energy density of the dipole for sufficiently small $\kappa$.

\subsection{The internal wave wake}

The propagating dipole appears in the outer field as a source doublet of strength $\mu$ propagating to the right at speed $U$. The wave drag on the dipole is weak and so over times of order of the advection time, the time taken for the internal wave wake to be established in the neighbourhood of the dipole, the dipole radius and speed are unaltered. The wave field can be thus taken as steady in the frame moving with the dipole. The nonlinear terms are negligible in (2.1), the material derivative is simply $-U \partial_{x}$, and the governing equations again reduce to (2.5) showing, incidentally, that the wavefield derived here satisfies the full nonlinear steady equations. For sufficiently small $x, z$ the buoyancy term in (2.5) is negligible so the inner region of the internal wave field is irrotational, joining smoothly to the irrotational outer part of the LC-dipole solution (2.8). The linear solution for horizontally propagating dipoles in a weakly stably stratified flow has been solved in both two and three dimensions by Gorodtsov \& Teodorovich (1983). The solution for two dimensional flow is summarised here and that for three dimensions is simply quoted where needed in $\S 5$. Equation (2.5) is solved in $z>0$ subject to the boundary condition (2.10) and the solution smoothly extended into $z<0$ by taking $\psi$ to be odd in $z$. This gives the Fourier transform solution,

$$
\begin{aligned}
\psi(x, z) & =\frac{1}{2 \pi} \int_{-\infty}^{\infty} \widehat{\psi}(k, z) \exp (\mathrm{i} k x) \mathrm{d} k, \\
\text { where } \quad \widehat{\psi}(k, z) & =\pi U a^{2} \begin{cases}\exp \left[-\left(k^{2}-\kappa^{2}\right)^{1 / 2} z\right] & |k|>\kappa \\
\exp \left[-\mathrm{i} \operatorname{sgn}(k)\left(\kappa^{2}-k^{2}\right)^{1 / 2} z\right] & |k|<\kappa,\end{cases}
\end{aligned}
$$

and the sign in the second exponential has been chosen to give upward group velocity.

The drag $D$ exerted on a material surface at height $z$ is given by the component of the pressure force in the negative- $x$ direction. Let $\zeta\left(x, z_{0}\right)$ be the displacement from height 
$z_{0}$ of a particle originally at height $z=z_{0}$ far upstream. Then

$$
D=-\int_{-\infty}^{\infty} p \zeta_{x} \mathrm{~d} x
$$

This force is positive, corresponding to a drag on the material surface, when there is a positive upward flux of $x$-momentum. Substituting $w=-U \zeta_{x}$ from the definition of $\zeta$ and $p=\rho U u$ from $(2.1 a)$ gives

$$
\begin{aligned}
D & =\rho \int_{-\infty}^{\infty} u w \mathrm{~d} x=-\rho \int_{-\infty}^{\infty} \psi_{x} \psi_{z} \mathrm{~d} x=-\frac{\rho}{2 \pi} \int_{-\infty}^{\infty} \widehat{\psi}_{x} \widehat{\psi}_{z}^{*} \mathrm{~d} k \\
& =-\frac{1}{2} \pi \rho U^{2} a^{4}\left\{\int_{|k|>\kappa} \mathrm{i} k\left(k^{2}-\kappa^{2}\right)^{1 / 2} \exp \left[-2\left(k^{2}-\kappa^{2}\right)^{1 / 2} z\right] \mathrm{d} k+\int_{-\kappa}^{\kappa}-|k|\left(\kappa^{2}-k^{2}\right)^{1 / 2} \mathrm{~d} k\right\} \\
& =-\frac{1}{3} \pi \rho U^{2} a^{4}\left[\left(\kappa^{2}-k^{2}\right)^{3 / 2}\right]_{0}^{\kappa}=\frac{1}{3} \pi \rho U^{2} a^{4} \kappa^{3} .
\end{aligned}
$$

Here $(2.15 a)$ follows from Plancherel's theorem and * denotes complex conjugate. The first integrand in $(2.15 b)$ is odd in $k$ so the integral vanishes, as expected for evanescent waves, and the second integrand is even giving $(2.15 c)$. Waves are radiated both upwards and downwards from the dipole and so the total drag on the dipole is twice that given by $(2.15 c)$.

The vertical energy flux is given by the flux of pressure, as in (A 1),

$$
F=\int_{-\infty}^{\infty} w p \mathrm{~d} x=\int_{-\infty}^{\infty}\left(-U \zeta_{x}\right) p \mathrm{~d} x=U D,
$$

the work done by the drag force.

\subsection{The vortex centre}

As the flow evolves, dipole fluid escapes the boundary of the dipole, in particular from the neighbourhood of the rear stagnation point. The central regions of the vortices comprising the dipole maintain their integrity and so we follow $\mathrm{FH}$ and consider the vorticity contained within these regions, defining the instantaneous vortex centre at a given time as the point within the vortex in $z>0$ where the total streamfunction, $\Psi=-U z+\psi$, at that time attains its maximum. Consider a total streamline sufficiently close to the vortex centre that it is closed. Integrating the vorticity over the region enclosed by this streamline gives, from $(2.1 a)$ and $(2.1 b)$,

$$
\int \eta_{t} \mathrm{~d} x \mathrm{~d} z+\oint \eta(\boldsymbol{u} . \hat{\boldsymbol{n}}) \mathrm{d} s=\int b_{x} \mathrm{~d} x \mathrm{~d} z=\oint b \mathrm{~d} z,
$$

where $\mathrm{d} s$ is the element of length along the streamline. Integrating the buoyancy over the same region gives, from $(2.1 c)$,

$$
\int b_{t} \mathrm{~d} x \mathrm{~d} z+\oint\left(b+N^{2} z\right)(\boldsymbol{u} \cdot \hat{\boldsymbol{n}}) \mathrm{d} s=0 .
$$

As the velocity normal to the streamline is zero, the line integral on the left sides of $(2.17)$ and (2.18) vanish. Now allow the streamline value to increase towards the maximum so that the integration area converges on the vortex centre. Provided the maximum buoyancy perturbation at times $t>0$ lies at the vortex centre, as it does initially, then the buoyancy terms on the right side of (2.17) vanish faster than the vorticity term on the left to give in the limit that $\eta_{t}$ vanishes at the vortex centre: the instantaneous 


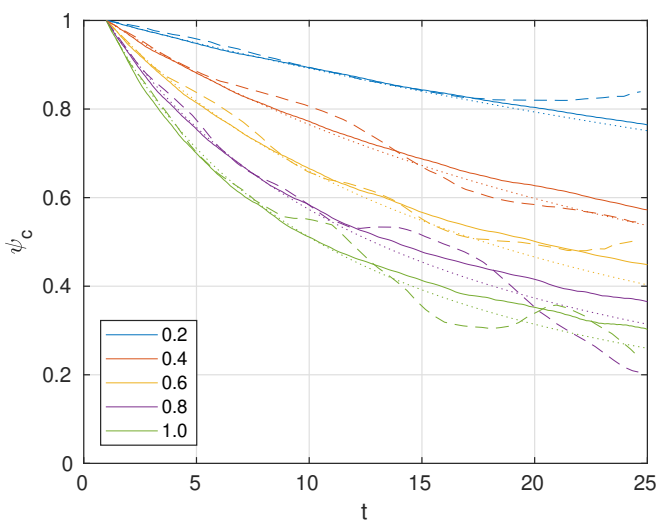

(a)

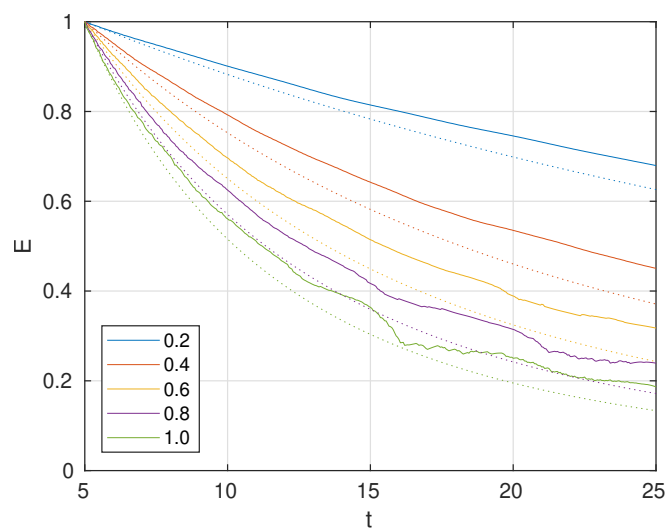

(b)

Figure 2: (a) The normalised maximum value of streamfunction within the quasigeostrophic modon: the streamfunction value at the vortex centre, $\psi_{c}$, and (b) the normalised modon energy, $E$, as functions of time, $t$ for the background vorticity gradients $\beta$ listed in the legend. The solid lines show the present computations, the dashed lines the computations of $\mathrm{FH}$ and the dotted lines the analytical predictions.

rate of change of the vorticity at the vortex centre is zero. The numerical simulations in $\S \S 3,4$ below confirm that the vorticity at the vortex centre, denoted herein by $\eta_{c}$ and described for brevity as the centre vorticity, remains approximately constant throughout the evolution. Similarly (2.18) shows that the perturbation buoyancy at the vortex centre, denoted herein by $b_{c}$ and described as the centre buoyancy, is constant throughout the evolution, again borne out by the numerical integrations of $\S 4$.

\section{Modon decay through Rossby wave radiation}

Before considering the slow evolution of the stratified flow it is useful to relate the analysis of $\S 2$ to that for the decay of modons due to Rossby wave radiation described by FH. The $\beta$-plane approximation consists of taking Cartesian axes $O x y$ in a horizontal plane with $O y$ northwards, corresponding velocity components $(u, v)$, streamfunction $\psi$ such that $(u, v)=\left(-\psi_{x}, \psi_{y}\right)$ and $\beta$ as the local poleward gradient of the vertical component of the Earth's rotation (figure 1b ). This gives the quasi-geostrophic potential vorticity equation in a frame moving at speed $U$ to the west, as in $\mathrm{FH}$, as

$$
\left(\partial_{t}-U \partial_{x}\right) \nabla^{2} \psi+\partial\left(\psi, \nabla^{2} \psi\right)+\beta \psi_{x}=0 .
$$

Equation (3.1) has the same dynamics as that in $\S 2$ on setting $\kappa=(-\beta / U)^{1 / 2}$ for $U<0$. The drag computation in $\S 2.3$ applies directly to give the same expression $(2.15 c)$ for the total drag due to Rossby wave radiation and (2.16) for the work done by the wavefield: the wave drag method of $\S 2$ gives the same results as the adjoint method of $\mathrm{FH}$.

Equating the rate of change of dipole impulse to the drag force acting on the dipole gives the slow evolution equation, taking into account that $U<0$,

$$
\frac{\mathrm{d} \mu}{\mathrm{d} t}=-2 D, \quad \text { i.e. } \frac{\mathrm{d}}{\mathrm{d} t}\left(2 \pi U a^{2}\right)=-\frac{2}{3} \pi U^{2} a^{4} \kappa^{3} .
$$

Similarly balancing the rate of change of vortex energy against the work done by the 


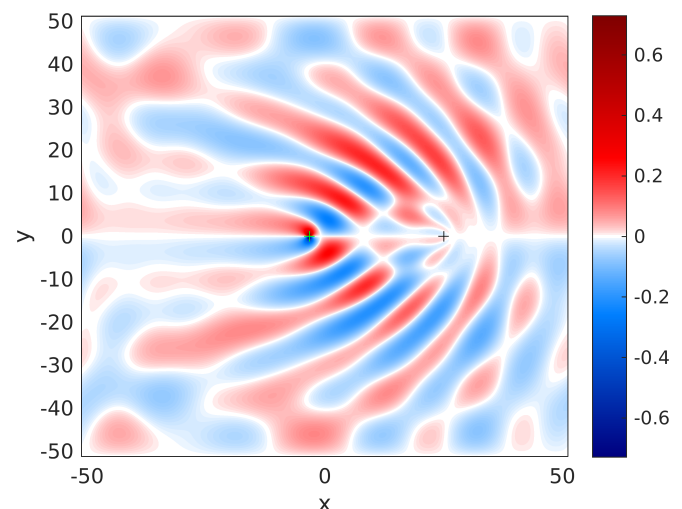

(a)

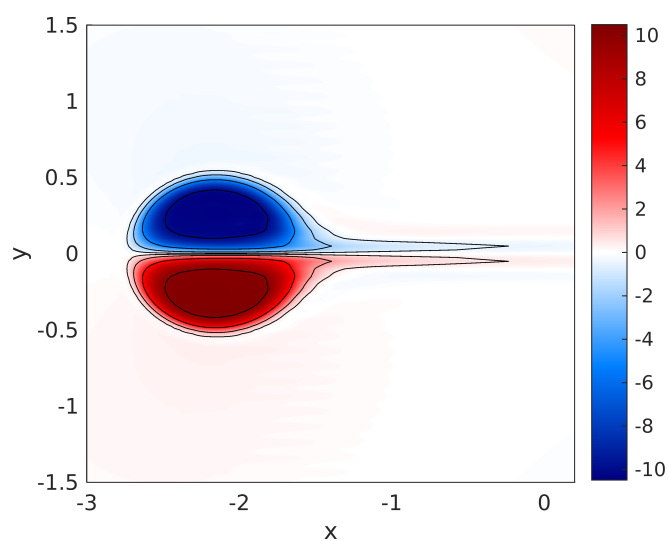

(b)

Figure 3: (a) The streamfunction $\psi$ in the horizontal $\beta$-plane forced by a westward propagating quasigeostrophic modon for $\beta=0.4$ at time $t=25$. The modon, marked by ' + ', moves west from an initial position marked with ' + ', generating the Rossby wave wake. (b) The relative vorticity $\nabla^{2} \psi$ for $\beta=0.8$ at $t=15$, showing vorticity escaping from the rear of the modon.

wavefield gives

$$
\frac{\mathrm{d} E}{\mathrm{~d} t}=-2 F=-2 U D, \quad \text { i.e. } \frac{\mathrm{d}}{\mathrm{d} t}\left(2 \pi U^{2} a^{2}\right)=-\frac{2}{3} \pi U^{3} a^{4} \kappa^{3} .
$$

Equations (3.2) and (3.3) combine to require that $U$ remains constant and so

$$
a=a_{0}\left(1+\frac{1}{3} \epsilon^{3}\left|U_{0}\right| t / a_{0}\right)^{-1 / 2}, \quad \epsilon=\epsilon_{\beta 0}=\left(-\beta a_{0}^{2} / U_{0}\right)^{1 / 2},
$$

where $a_{0}$ and $U_{0}$ are the initial values of $a$ and $U$. This is the same result that $\mathrm{FH}$ obtain by combining energy conservation with enstrophy conservation. They point out that the peak vorticity within the modon is proportional to $U / a$ and so would increase as the modon shrank, violating Lagrangian conservation of peak vorticity. FH note also that streamers of vorticity are shed from the rear of the dipole, consistent with the instability described by Brion et al. (2014), and so it is reasonable to expect that dipole enstrophy is not conserved. Enforcing the conservation of centre vorticity, defined as in $\S 2.4$ is equivalent to requiring that $U / a$ remains constant throughout the evolution. Requiring $U / a$ to remain equal to its initial value while relaxing conservation of enstrophy or, equivalently, the drag - modon impulse balance (3.2), and retaining the work - modon energy balance (3.3), gives the decay rate

$$
a_{1}(t)=a_{0}\left(1+\frac{1}{8} \epsilon^{3}\left|U_{0}\right| t / a_{0}\right)^{-2 / 3} .
$$

FH present numerical integrations that strongly support the determination through conservation of centre vorticity. The agreement of the decay rate of various quantities with (3.5) is excellent, particularly in the relevant range of small $\beta$, even though the accompanying wavefield seems not to take precisely the asymptotic form. This deviation and the large fluctuations at larger $\beta$ appear to be due to the necessarily finite size of the computational domain. To test this hypothesis equation (3.1) was discretized spectrally using Fourier series on a doubly periodic domain as in $\mathrm{FH}$, but with twice the resolution and four times the domain size $(2048 \times 2048$ points on a grid of size $102.4 \times 102.4$ initialised with an LC-dipole vortex of radius one and speed one) and integrated using the Dedalus package (Burns et al. 2020) with a timestep of $2 \times 10^{-3}$ and hyperdiffusion included to 
absorb the downscale enstrophy cascade and ensure numerical stability. The total energy in the domain was conserved to within $1 \%$ over 25 time units for all $\beta$ considered and the centre vorticity to within $5 \%$. The position of the maximum streamfunction value, $\psi_{c}(t)$, i.e. the vortex centre, is known accurately at each time step and so the instantaneous speed $U(t)$ can be obtained by differencing the $x$-coordinate of the vortex centre. The instantaneous dipole radius $a(t)$ is more difficult to measure directly. The assumption that the dipole evolves to remain an LC-dipole means however, from (2.8), that

$$
\psi_{c}(t)=P a(t) U(t)
$$

for some constant $P$. By direct evaluation of the maximum of $(2.8), \mathrm{FH}$ show that $P \approx 1.3$ and so $a(t)$ follows from (3.6). The initial dipole adjusts rapidly at early times altering slightly both the initial speed and radius of the modon. We follow $\mathrm{FH}$ and evaluate $a_{0}$ and $U_{0}$ from the flow field at some time $t=t_{0}$ after this initial adjustment. These values then determine $\epsilon$ through (3.4), which thus differs slightly from $\beta^{1 / 2}$. For definiteness the integrations are labelled by the value of $\beta$.

Figure 2(a) compares the present results for the maximum value of the streamfunction within the modon, the streamfunction value at the vortex centre as defined in $\S 2.4$, with those of $\mathrm{FH}$ showing that decreasing the effect of westward-radiated waves re-entering from the eastern boundary, and decreasing wave reflection at the zonal boundaries, due to spatial periodicity, suppresses the fluctuations present on the smaller computational domain. Figure 2(b) shows the decay of modon energy $E$, calculated here as the kinetic energy within a circle of radius $a(t)$ about the translating origin of the dipole. The small $\beta$ results capture the qualitative behaviour even for $\beta=1$ where the modon has lost almost $80 \%$ of its energy by $t=25$. The values for the determination for $a_{0}$ and $U_{0}$ are $t_{0}=1$ in (a) and $t_{0}=5$ in (b) as the streamfunction appears to fall into its asymptotic behaviour sooner. The values plotted have been normalised by their value at $t=t_{0}$.

Figure 3(a) shows the wavefield at time $t=25$ for $\beta=0.4$ in an inertial frame propagating to the west at unit speed. The initial modon position is marked by ' + ' (and thus is at $X=x^{\prime}+t=25$ at $t=25$ ) and the current position by ' + '. The wake behind the modon is established between the initial and current modon positions as the modon moves away. Slowly propagating transient waves can be seen originating from the neighbourhood of the initial modon position. As noted by $\mathrm{FH}$, the modon initially accelerates through the $\beta$-effect to move slightly faster than unity but then slows due to wave drag and so by $t=25$ lies just in $X<0$. The wavefield has the expected asymptotic form with no clear signature of boundary effects. These features of the solution can be seen more clearly in the movie (Movie1.mp4) of the temporal development of $\psi$, included as supplementary material. Figure 3(b) shows the vorticity expelled from the rear of the modon as expected from the LC-dipole stability analysis of Brion et al. (2014) and from the viscous LC-dipole computations of Nielsen \& Rasmussen (1997).

\section{The slow evolution of the stratified flow}

The equations corresponding to (3.2) and (3.3) for stratified flow, where $\kappa=N / U$ are the impulse-drag equation

$$
\frac{\mathrm{d}}{\mathrm{d} t}\left(U a^{2}\right)=-N^{3} a^{4} / 3 U .
$$

and the energy-work equation

$$
\frac{\mathrm{d}}{\mathrm{d} t}\left(U^{2} a^{2}\right)=-N^{3} a^{4} / 3 .
$$




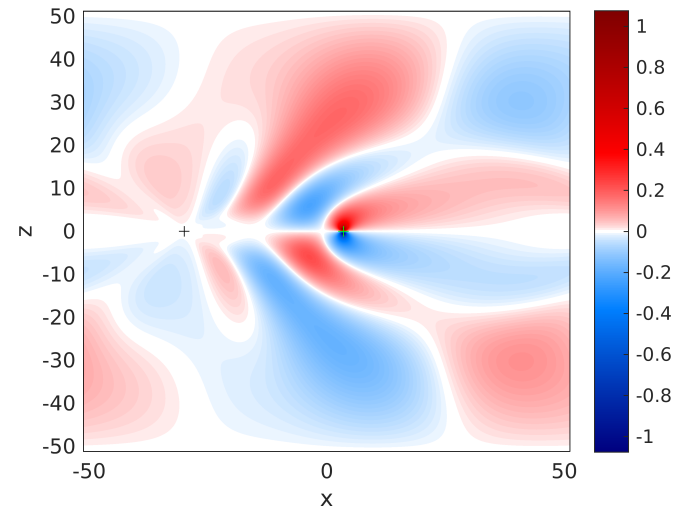

(a)

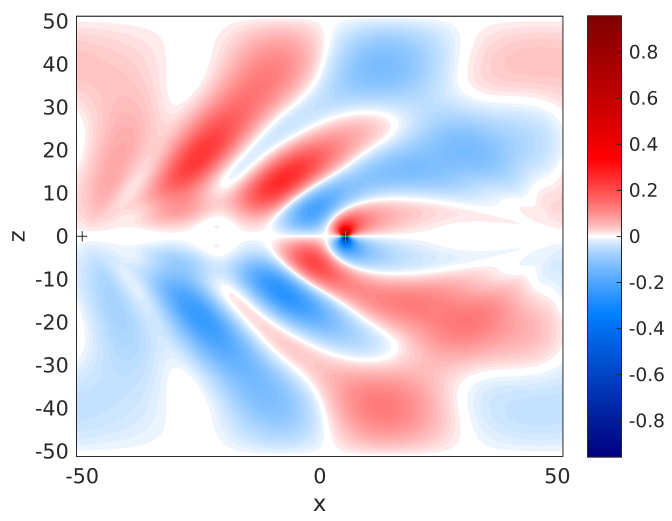

(b)

Figure 4: The streamfunction $\psi$ for a rightward propagating dipole in the vertical-slice stratified model for $N=0.4$ at times (a) $t=30$, (b) $t=50$. The dipole, marked by ' + ', moves to the right from an initial position marked with ' + ' , generating the internal wave wake.

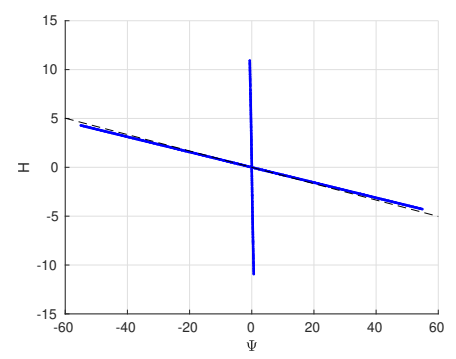

(a)

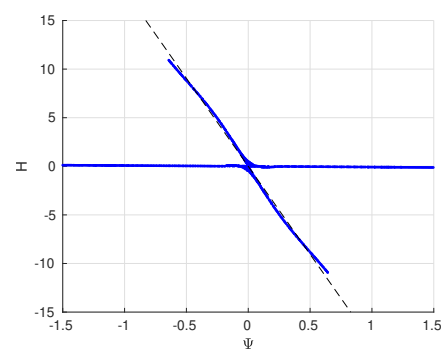

(b)

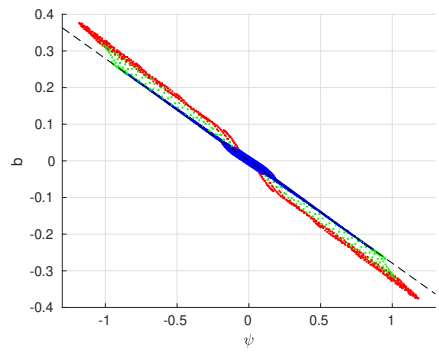

(c)

Figure 5: Scatter plots showing the functional relations in the flow at time $t=30$ for buoyancy $N=0.4$. (a) The total vorticity $H=\left(N^{2} / U\right) z+\nabla^{2} \psi$ as a function of of the total streamfunction $\Psi=-U z+\psi$ over the whole flow field. (b) A detail of part (a) in the neighbourhood of the dipole. (c) The perturbation buoyancy $b$ as a function of the perturbation streamfunction $\psi$ over the whole flow field. The dashed lines in each part give the theoretical relations discussed in the text.

Again equations (4.1) and (4.2) combine to require that $U$ remains constant, giving (3.4) with here $\epsilon=\epsilon_{N 0}=N a_{0} / U_{0}$, and so contradicting the conservation of centre vorticity of $\S 2.4$. Relaxing the dipole impulse equation (4.1) while requiring the centre vorticity to be conserved, i.e. $U / a$ to remain constant, and the energy equation (4.2) to hold, gives

$$
a_{1}(t)=a_{0} \exp \left(-\frac{1}{12} \epsilon_{N}^{3}\left|U_{0}\right| t / a_{0}\right) .
$$

The decay rate is exponential here in contrast to the slower algebraic decay rate for the $\beta$ plane modon because of the different power of $U$ in the definition of $\kappa$. The conservation of centre vorticity also implies that the instantaneous value of the inverse Froude number, $\epsilon_{N}=\kappa a=N a / U$, remains equal to its initial value, $\epsilon_{N 0}$, in contrast to the $\beta$-plane modon where the instantaneous value of $\epsilon_{\beta}$ slowly decreases with time, as $a^{1 / 2}$, and so the flow becomes progressively less nonlinear. As expected from $\S 2$ the decay timescale is of order $\epsilon^{-3}$ times the advection timescale.

Equations (2.1) in streamfunction-vorticity form, i.e. the unsteady version of equations 
(2.3), were integrated using the code of $\S 3$, including the additional buoyancy field, with the initial condition for the vorticity taken to be an LC-dipole, the initial streamfunction obtained from the vorticity by inverting the periodic Laplacian, and the initial buoyancy to be given by $(2.5 b)$ so as to be continuous and have continuous normal derivative at $r=a$. The flow pattern developed more slowly than on the $\beta$-plane and so the equations were integrated to $t=50$, with the total energy again conserved to within $1 \%$ over all integrations. The dipoles again evolve slightly but rapidly initially and so $a_{0}$ and $U_{0}$ are computed at time $t=t_{0}$ after this initial evolution with the integrations labelled by the value of $N$.

Figure 4 shows the wavefield for $N=0.4$ at times $t=30$ and $t=50$ in an inertial frame propagating to the right at unit speed. The initial dipole position is marked by ' + ' (and thus is at $X=x^{\prime}-t=-30$ at $t=30$ and at $X=-50$ at $\left.t=50\right)$ and the current position by ' + '. As for the $\beta$-plane modon of $\S 3$, the wake behind the dipole is set up between the initial and current dipole positions as the dipole moves away; slowly propagating transient waves can be seen originating from the neighbourhood of the initial modon position; and the dipole initially accelerates, here through buoyancy effects, to move slightly faster than unity but then slows due to wave drag and so lies just in $X>0$ at $t=30,50$. The wavefields are well developed, particularly in the neighbourhood of the dipole, boundary effects are negligible, and the wavelength of the disturbance remains constant with time, as expected. These features can be seen more clearly in the movie (Movie2.mp4) of the temporal development of $\psi$ and $b$, included as supplementary material.

The model here assumes that throughout the evolution the dipole remains an LCdipole, though with varying speed and radius, and that the streamfunction and buoyancy are linearly related. Figure 5 tests these relations at time $t=30$ for $N=0.3$ for the $4 \times 10^{6}$ computational points. Figure 5(a) gives a scatter plot of the vorticity $H=\left(N^{2} / U\right) z+\nabla^{2} \psi$ as a function of the total streamfunction $\Psi=-U z+\psi$ over the whole flow field and figure 5(b) gives a detail of 5(a) in the neighbourhood of the dipole. From (2.5a) and (2.7) the points outside the dipole should lie along a line through the origin with slope $\kappa^{2}$ and points inside the dipole along a line with slope $K^{2}-\kappa^{2}$, shown dashed in $5(\mathrm{a})$ and (b). The closeness of the computed values even at large times shows that the dipole remains close to an LC-dipole. A scatter plot of the total density against the total streamfunction (not shown here) shows that these quantities are very closely linearly related, satisfying $(2.3 b)$ closely. A more stringent test is shown in figure 5 (c) where the perturbation buoyancy $b$ is plotted as a function of the perturbation streamfunction $\psi$ over the whole flow field. The general behaviour accords with the relations $(2.5 b)$ and $(2.7)$, with the slope of the theoretical relations reproduced accurately. The blue points correspond to computational points lying outside the dipole and lie accurately along the line of $(2.5 b)$. The red points are points lying within the dipole. They show a slightly steeper slope near the geometric centre of the dipole at the co-moving-coordinate origin and a small offset over the majority of the dipole. The points near the ends of the red groups correspond to computational points near the vortex centre. This behaviour cannot be captured by the model presented here but appears to be sufficiently small that it does not alter the qualitative behaviour of the flow. This will be discussed briefly in $\S 7$. The green points are computational points near the dipole boundary affected by the computational hyperdiffusion which smooths otherwise discontinuous higher gradients there.

Figure 6 shows the centre vorticity $\eta_{c}$ and the centre perturbation buoyancy $b_{c}$ as functions of time $t$ for different strengths of the background buoyancy gradient, $N$. The values are normalised on their value at $t=t_{0}$ where here $t_{0}=10$, reflecting the longer time required for the stratified dipole to adjust to its asymptotic form. For small $N, \eta_{c}$ is closely constant and even for $N=0.4, \eta_{c}$ decreases only by approximately $1.5 \%$ over 


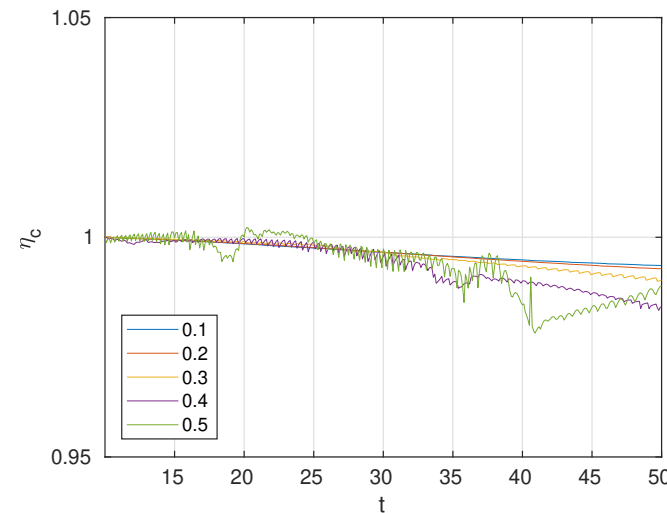

(a)

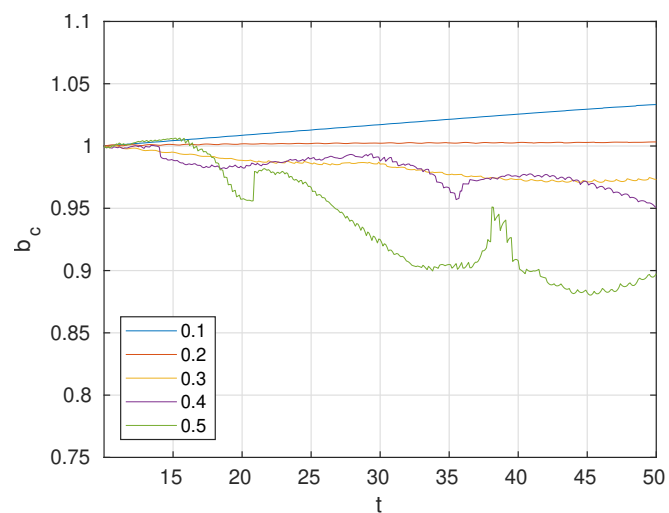

(b)

Figure 6: The normalised maximum value of (a) the centre vorticity, $\eta_{\mathrm{c}}$, and (b) the centre perturbation buoyancy, $b_{\mathrm{c}}$, as functions of time, $t$ for the values of the background buoyancy frequency, $N$, listed in the legend.

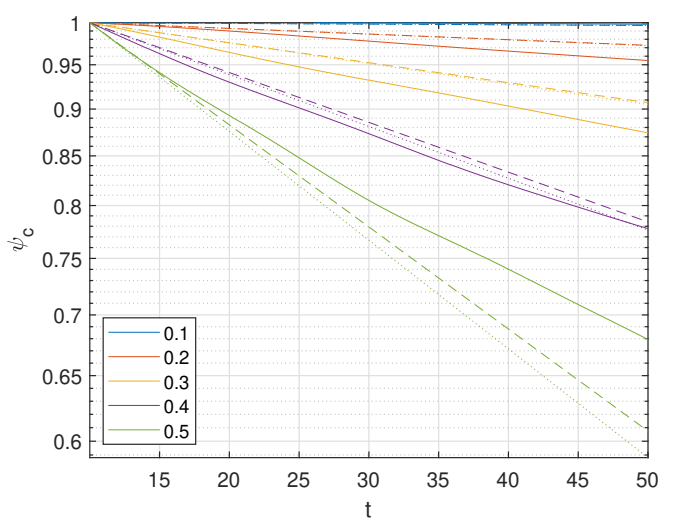

(a)

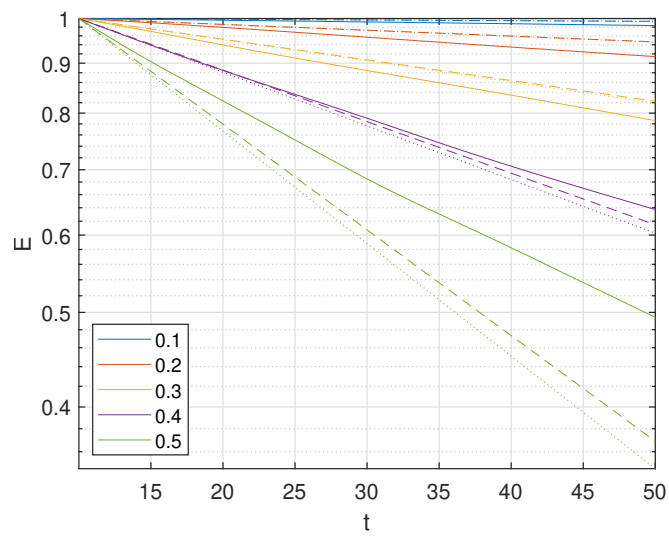

(b)

Figure 7: Normalised values of (a) the maximum value of the streamfunction $\psi_{\mathrm{c}}$ at the vortex centre and (b) the dipole energy $E$ as functions of time $t$ for the values of the buoyancy frequency $N$ listed in the legend. The axes are log-linear. Solid lines give the values from the numerical integrations, dotted straight lines those from the dipole theory and dashed straight lines the values from the cylindrical approximation.

the integration. The centre perturbation buoyancy, $b_{c}$, remains within $3 \%$ of its value at $t=t_{0}$ provided $N \leqslant 0.3$. The buoyancy fluctuations for larger $N$ appear related to stirring at the vortex centre, discussed briefly in $\S 7$. Figure 7 shows the streamfunction $\psi_{\mathrm{c}}$ at the vortex centre and the dipole energy $E$ during the dipole evolution and figure 8 (a) the dipole impulse $\mu$, normalised by their values at $t=t_{0}=10$. The dipole model results appear to capture the decay here with comparable accuracy to $\S 3$ even over the longer integration time.

Gorodtsov \& Teodorovich (1983) point out that approximating a finite body by a dipole overestimates the drag by including finite contributions from arbitrarily large wavenumbers which are not forced by the body. By approximating a cylinder of radius $a$ by a distributions of sources Gorodtsov \& Teodorovich (1983) obtain the drag formula, 


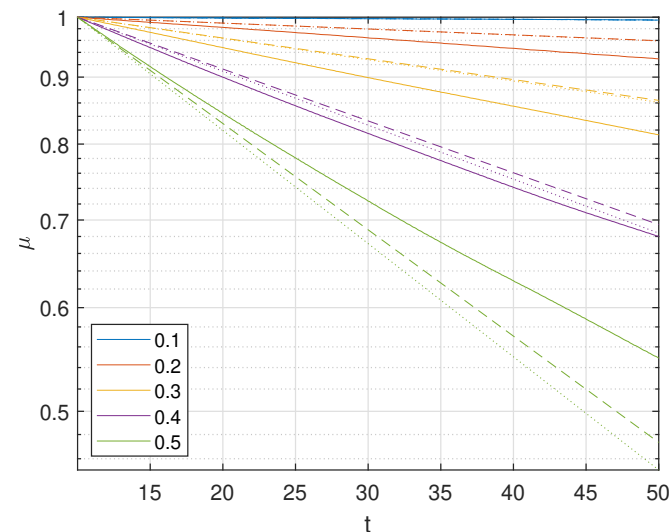

(a)

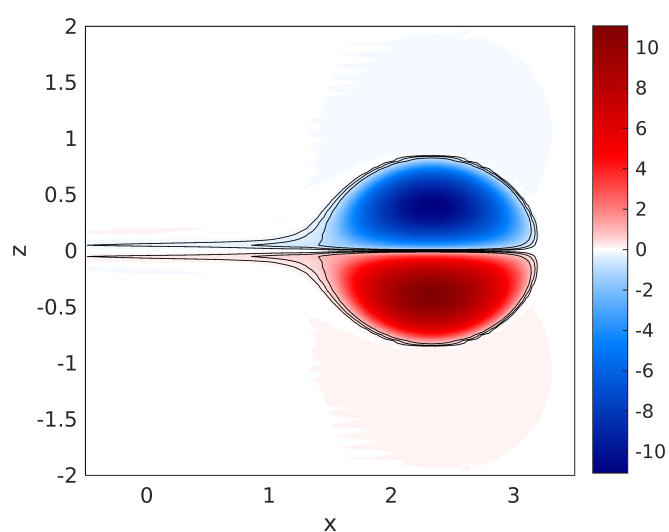

(b)

Figure 8: (a) As in figure 7 but for the dipole impulse $\mu$. (b) The relative vorticity $\eta=\nabla^{2} \psi$ in a vertical $(x, z)$ slice for $N=0.5$ at time $t=15$, showing vorticity escaping from the rear of the dipole.

in the notation of $\S 2.3$,

$$
D_{C}=(8 / 3) \pi \rho U^{2} a^{2} \kappa\left[\mathrm{J}_{1}(\kappa a)\right]^{2},
$$

which they argue is a more accurate estimate of the wave drag as it takes into account the finite size of the forcing region. Substituting $U D_{C}$ for the work done by the vortex on the fluid in the work-energy equation and imposing conservation of centre vorticity then gives the modified decay formula

$$
a_{2}(t)=a_{0} \exp \left(-\frac{1}{3} \epsilon_{N}\left[\mathrm{~J}_{1}\left(\epsilon_{N}\right)\right]^{2}\left|U_{0}\right| t / a_{0}\right) .
$$

Expression (4.5) reduces to (4.3) in the limit $\epsilon_{N} \rightarrow 0$ and is not correct to any higher order in $\epsilon_{N}$ than (4.3). To assess whether accounting for the finite vortex size through this cylindrical approximation gives a more accurate model than the dipole model at small but finite $\epsilon_{N}$ the values of $\psi_{c}, E$ and $\mu$ derived from (3.5) are included in figures 7 and 8 (a) as dashed lines. The cylindrical approximation gives a slower decay as expected but the effect for these values of $N$ is negligible compared with the effect of unavoidable small variations in the estimation of $a_{0}$ and $U_{0}$ and so finite size effects are not the leading order omitted effect for stratified dipoles. The same argument applies to the modon decay of $\S 3$ but since the instantaneous inverse Froude number, $\epsilon_{\beta}=\kappa a=\left(-\beta a^{2} / U\right)^{1 / 2}$, decreases during the evolution the simple form of solution (3.5) is lost. Approximating the Bessel function in (4.4) by its first two terms gives the radius decay, with the first correction, as

$$
a_{2}(t)=a_{0} \tau\left[1+\frac{3}{8} \epsilon_{\beta 0}^{2}\left(1-\tau^{2}\right)\right], \quad \tau=\left(1+\frac{1}{8} \epsilon_{\beta 0}^{3}\left|U_{0}\right| t / a_{0}\right)^{-2 / 3} .
$$

As for (4.5), (4.6) reduces to (3.5) in the limit $\epsilon_{\beta 0} \rightarrow 0$ and is not correct to any higher order in $\epsilon_{\beta 0}$ than (3.5). Comparison with the dipole model (not plotted here) show again that finite size effects are not the leading order omitted effect for $\beta$-plane modons.

Figure 8 (b) shows the relative vorticity $\eta=\nabla^{2} \psi$ in a vertical $(x, z)$ slice for buoyancy $N=0.5$ at time $t=15$. As for the $\beta$-plane modon, vortical fluid escapes from the rear of the dipole as expected from the LC-dipole stability analysis of Brion et al. (2014) and from the viscous LC-dipole computations of Nielsen \& Rasmussen (1997). 


\section{Other geometries}

\subsection{A cylindrical dipole in a rotating frame}

The correspondence between two-dimensional rotating and stratified flows noted by Bretherton (1967) means that the results above apply directly to rotating flow. Consider the geometry of $\S 2$ with a LC-dipole of radius $a$ and axis aligned with the $y$ axis advancing in the $x$-direction with speed $U$ and take the fluid to be of constant density but rotating at angular velocity $\Omega$ about the $x$-axis (figure 1c). The governing equations in a frame moving with the vortex can then be written

$$
\left(\partial_{t}-U \partial_{x}\right) \boldsymbol{u}+2 \Omega \hat{\boldsymbol{x}} \times \boldsymbol{u}=-(1 / \rho) \nabla p, \quad \nabla \cdot \boldsymbol{u}=0 .
$$

Writing $b=-2 \Omega v$ and $\mathrm{N}=2 \Omega$ reduces (5.1) to (2.1). The results for a stratified flow carry over directly on taking $\epsilon=\epsilon_{\Omega}=2 \Omega a_{0} / U_{0}$, the inverse Rossby number, to be small. The internal wave field of the stratified flow becomes a large-scale, weak, inertial wave wake. The buoyancy field maps to the cross-flow velocity field, $v$, in the $y$-direction.

\subsection{Hill's vortex in a stratified fluid}

The model put forward in $\S 2$ extends straightforwardly to three dimensions. Let the nonlinear inner dipole be the Hill's spherical vortex of radius $a$ advancing in the $x$ direction with speed $U$ (figure 1d). As with the LC-dipole this gives an inner vortical flow surrounded by irrotational flow. The energy of the vortex is $10 \pi \rho a^{3} U^{2} / 7$ (Lamb 1932, Art. 165). For small inverse Froude number, $\epsilon_{N}$, the outer field is the internal wave field behind a sphere of radius $a$ moving at speed $U$. This field exerts a drag on the sphere equal to, in the notation of $\S 2.3, D_{S}=(\pi / 8) \rho a^{4} N^{2} \epsilon_{N}^{2}\left(-\log \epsilon_{N}+7 / 4-\gamma\right)$, where $\gamma \approx 0.577$ is Euler's constant, (Gorodtsov \& Teodorovich 1983) doing work at a rate $U D_{S}$. The energy-work-done equation is thus

$$
\frac{\mathrm{d}}{\mathrm{d} t}\left(U^{2} a^{3}\right)=-(7 / 80) U N^{2} a^{4} \epsilon_{N}^{2}\left(-\log \epsilon_{N}+7 / 4-\gamma\right) .
$$

Lamb (1932, Art. 165) notes that the conserved scalar vortical quantity is the azimuthal (around the $x$-axis) component vorticity divided by distance from the $x$-axis. The argument of $\S 2.4$ then shows that the vortex centre value of the azimuthal vorticity is constant throughout the motion, and so $U / a$ and the instantaneous value of $\epsilon_{N}$ remain constant. Combining this with (5.2) gives a predicted decay for the vortex radius as

$$
a(t)=a_{0} \exp \left[-(7 / 400) \epsilon_{N}^{4}\left(-\log \epsilon_{N}+7 / 4-\gamma\right) U_{0} t / a_{0}\right] .
$$

Similarly to $\S 2$, a pressure perturbation of order $\epsilon_{N}^{2}$ acting over an area of order $\epsilon_{N}^{2}$ gives a decay timescale of order $\epsilon_{N}^{-4}$ times the advection timescale.

The model here also extends to the motion of Hill's vortex along the axis of a weakly rotating fluid although the axisymmetric geometry introduces technical differences in both the analytical and numerical methods (Crowe et al. 2021).

\section{Viscous dissipation}

Viscosity and density diffusion are present in real fluids. For the weak stratification limit considered here the leading order effect of these, for Prandtl and Schmidt numbers of order unity or greater, is the viscous dissipation of the LC-dipole. The Navier-Stokes equations for an incompressible fluid give the dissipation rate of the kinetic energy as $\nu \rho \mathcal{E}$ where $\nu$ is the kinematic viscosity, $\mathcal{E}=\int \eta^{2} \mathrm{~d} V$ is the enstrophy, and the integral is taken over a region sufficiently large that the vorticity is vanishingly small on its surface 


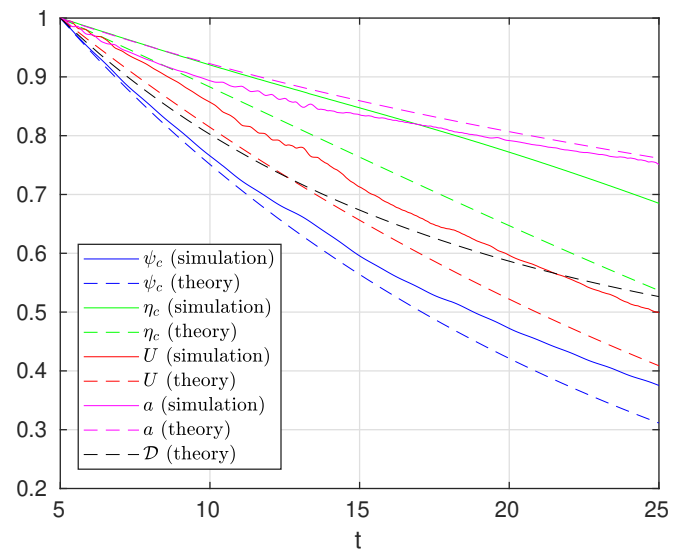

(a)

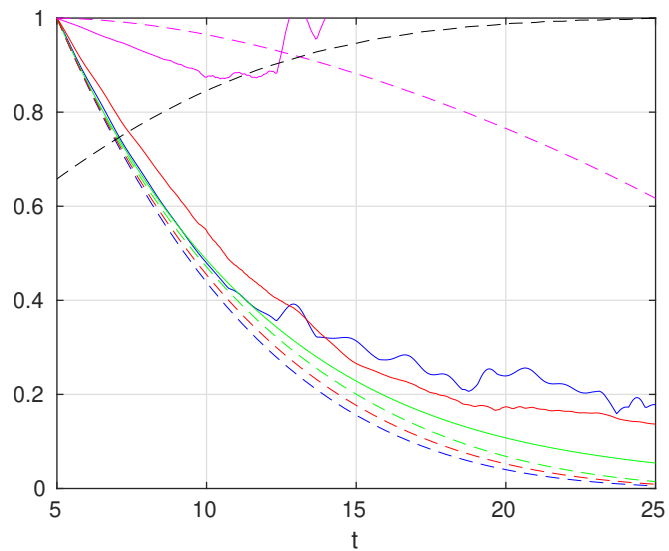

(b)

Figure 9: Simulation and predicted values for the maximum streamfunction, $\psi_{\mathrm{c}}$, centre vorticity, $\eta_{\mathrm{c}}$, vortex speed $U$ and vortex radius $a$, normalised on their initial values, and the dispersion/viscosity ratio $\mathcal{D}$ normalised on its maximum value, for a $\beta$-plane modon with $\beta=0.6$. (a) $\nu=0.001$, giving a value at $t=t_{0}=5$ of $\mathcal{D} \approx 178$. (b) $\nu=0.01$ giving a value at at $t=t_{0}$ of $\mathcal{D} \approx 45$. Legend as in (a).

(e.g. Nielsen \& Rasmussen 1997). Retaining the two leading order small effects for a weakly viscous, weakly stratified flow in the energy equation (A 3) gives

$$
\frac{\mathrm{d} E}{\mathrm{~d} t}=-\oint_{C} p \boldsymbol{u} \cdot \hat{\boldsymbol{n}} \mathrm{d} s-\nu \rho \mathcal{E} .
$$

For the LC-dipole, (2.8) gives $\mathcal{E}=K^{2} E$ and so, as in (3.3) and (4.2),

$$
\frac{\mathrm{d}}{\mathrm{d} t}\left(U^{2} a^{2}\right)=-\left(U^{3} a / 3\right)\left(\epsilon^{3}+3 \gamma^{2} \operatorname{Re}^{-1}\right),
$$

where $\epsilon=a \kappa$ is the inverse Froude number and $\operatorname{Re}=U a / \nu$ is the Reynolds number. The importance of wave drag relative to dissipation, for small $\epsilon$ here, is measured by the parameter $\mathcal{D}=\epsilon^{3}$ Re. The two effects are of the same order in (6.2) when $\mathcal{D} \approx 3 \gamma^{2} \approx$ 44 ; when $\mathcal{D} \gg 1$ wave drag dominates the decay and when $\mathcal{D} \lesssim 1$ viscous dissipation dominates. The stratified dipole, with $\mathcal{D}=N^{3} a^{4} / \nu U^{2}$, is more sensitive to dipole speed than the $\beta$-plane modem where $\mathcal{D}=\beta^{3 / 2} a^{4} / \nu(-U)^{1 / 2}$. As $a$ and $U$ evolve the value of $\mathcal{D}$ changes and the dominant decay mechanism can change.

van de Fliert (1996) combines the dispersionless $(\epsilon=0)$ version of $(6.2)$ with conservation of the impulse $\mu$ and Nielsen \& Rasmussen (1997) combine the dispersionless version of (6.2) with an equivalent equation for the viscous decay of enstrophy. Both derive equivalent models where the LC-dipole slows with speed $U \sim t^{-1}$ but radius expanding with $a \sim t^{1 / 2}$. These match closely numerical results in Nielsen \& Rasmussen (1997) although subsequent longer integrations (van Geffen \& van Heijst 1998; Delbende \& Rossi 2009) differ more due to the dipole structure deviating from the LC-dipole form. Neither closure is suitable for the dispersive problem. Section 4 shows that including the dispersive impulse-drag equation leads to the erroneous prediction of constant vortex propagation speed $U$, as does the inclusion of dispersive enstrophy decay on the $\beta$ plane, shown by FH. This suggests considering rather the dissipation of centre vorticity, $\eta_{c}$. Applying the argument of $\S 2.4$ shows that the advective contribution to temporal 


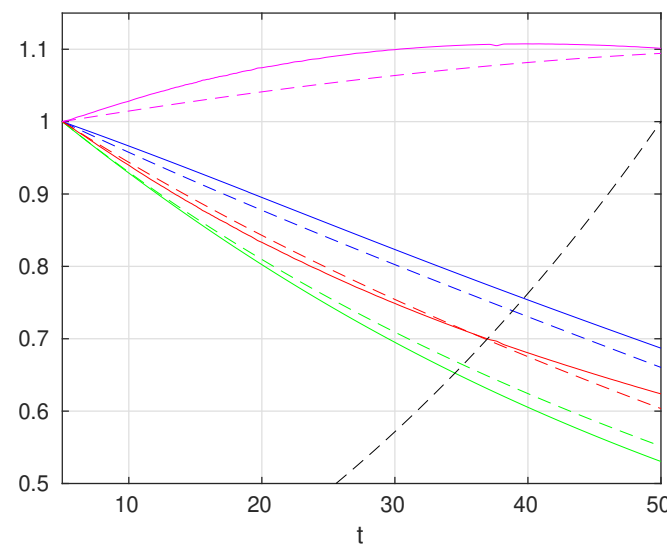

(a)

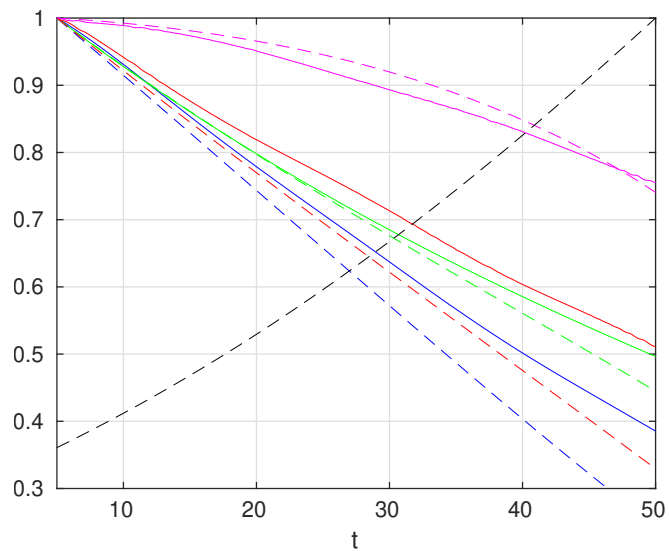

(b)

Figure 10: As for figure 9, but for a stratified dipole with $\nu=0.001$ (a) $N=0.2$, giving a value at $t=t_{0}=5$ of $\mathcal{D} \approx 8$. (b) $N=0.4$, giving a value at at $t=t_{0}$ of $\mathcal{D} \approx 58$. Legend as in figure 9 .

changes to $\eta_{c}$ vanishes, giving the vorticity diffusion equation

$$
\left(\eta_{t}-\nu \nabla^{2} \eta\right)_{c}=0
$$

For the hypothesised LC-dipole form (2.8) this gives

$$
\frac{\mathrm{d}}{\mathrm{d} t}(U / a)=-\gamma^{2} \nu U / a^{3} .
$$

Closing the specification of $a$ and $U$ by combining (6.2) and (6.4) gives a model that reproduces the results of $\S 3$ and $\S 4$ in the inviscid limit, $\nu=0$. In the dispersionless limit, $\epsilon=0,(6.2)$ and (6.4) give

$$
a(t)=a_{0}\left(1+\gamma^{2} \nu t / 2 a_{0}^{2}\right)^{1 / 2}, \quad U(t)=U_{0}\left(1+\gamma^{2} \nu t / 2 a_{0}^{2}\right)^{-3 / 2} .
$$

These formulae fit the numerical results in Nielsen \& Rasmussen (1997) and van Geffen \& van Heijst (1998) reasonably well, deviating most in the prediction for $a$ where, although (6.5) has the correct $t^{1 / 2}$ behaviour, the coefficient is too small. For general $\epsilon$ and $\nu$, the presence of two effects in (6.2) appears to preclude a simple analytical solution for the model but solutions are smooth and so can be obtained rapidly by standard numerical integration.

Figure 9 (a) shows the maximum streamfunction, $\psi_{\mathrm{c}}$, centre vorticity, $\eta_{\mathrm{c}}$, vortex speed $U$ and vortex radius $a$ (estimated as $\psi_{\mathrm{c}} / U$ ) from numerically integrating the $\beta$-plane equations, as in $\S 3$, with the inclusion of Navier-Stokes dissipation. Here $\beta=0.6$ and $\nu=0.001$. The initial adjustment of the vortex appears complete by $t=t_{0}=5$ when $U_{0} \approx 1.15$ and $a_{0} \approx 0.8$, giving an initial dispersion/viscosity ratio of $\mathcal{D} \approx 178$. The accuracy of the predictions from integrating equations (6.2) and (6.4) is comparable to that for inviscid flow in $\S 3$. Viscous effects become relatively stronger as the vortex slows with $\mathcal{D}$ decreasing to $\mathcal{D} \approx 90$ by $t=25$, when the dipole has lost $90 \%$ of its initial energy. Figure 9 (b) shows the equivalent simulation for $\nu=0.01$. This gives an initial value of $\mathcal{D} \approx 45$ and the stronger viscous effects cause the dipole to decay far more rapidly, losing $90 \%$ of its initial energy by $t \approx 12$, and subsequently deviating from the LC-dipole form.

Figure 10 shows the quantities of Figure 9 for a stratified dipole from numerically integrating as in $\S 4$, including Navier-Stokes dissipation with $\nu=0.001$. In figure 10 (a), 
where $N=0.2$, the initial adjustment appears complete by $t=t_{0}=5$ when $U_{0} \approx 1$ and $a_{0} \approx 1$, giving an initial dispersion/viscosity ratio of $\mathcal{D} \approx 8$. Viscous diffusion dominates the flow initially and the dipole expands. However the strong dependence of $\mathcal{D}$ on $U$ in stratified flow means that $\mathcal{D}$ increases rapidly as the dipole slows so by $t=50$, when the dipole has lost $45 \%$ of its initial energy, $\mathcal{D} \approx 32$, wave drag contributes comparably and the dipole ceases to expand. Figure 10(b) shows the equivalent simulation for $N=0.4$. This gives an initial value of $\mathcal{D} \approx 58$ so wave drag is stronger and the dipole decays more rapidly, losing $95 \%$ of its initial energy by $t=50$, when $\mathcal{D} \approx 161$. The accuracy of the predictions from integrating equations (6.2) and (6.4) is comparable to that for inviscid flow in $\S 4$, capturing in particular the viscosity-induced decay of the centre vorticity, $\eta_{\mathrm{c}}$.

\section{Discussion}

A model for the decay of dipolar vortices in weakly dispersive, inviscid, diffusionless flows has been put forward based on the hypothesis that the vortex remains a LCdipole, but of varying radius and speed, during its evolution. The decay is estimated here from balancing the loss of dipole energy against the work done by wave drag and using the conservation of centre vorticity, $\eta_{c}$, based on an argument of FH. For a $\beta$ plane modon the results reproduce those obtained by $\mathrm{FH}$ using an adjoint analysis. Numerical integrations of the full equations show reasonable agreement with the model predictions both for the $\beta$-plane flows and in a vertical-slice stratified flow. Figures 5 (c) and 6 (b) show that the leading order effect omitted from the stratified model appears to be the inviscid stirring of density within the vortex. The perturbation density remains a function of the perturbation streamfunction but is displaced a small but finite amount at finite $N$. Ford et al. (2000) discuss higher-order surface-wave effects on the advection of potential vorticity and it appears that similar higher order effects on the advection of dipole buoyancy contribute here.

The model is extended to weakly viscous flows in $\S 6$ by including viscous dissipation of vortex energy and viscous dissipation of centre vorticity. In the dispersionless limit this model approximates the results of van de Fliert (1996) and Nielsen \& Rasmussen (1997) for a homogeneous fluid but unlike their determinations, based on impulse conservation and enstrophy decay respectively, also reproduces the inviscid, dispersive results. The relative importance of the two effects, for small $\epsilon$ here, is measured by $\mathcal{D}=\epsilon^{3} \operatorname{Re}$ : when $\mathcal{D} \gg 1$ wave drag dominates and when $\mathcal{D} \lesssim 1$ viscous dissipation dominates.

The effect on the radiated wavefield of finite vortex size has been shown to be negligible compared to inaccuracies in the estimation of the initial dipole radius $a_{0}$ and speed $U_{0}$. The dipole moments of the vorticity and density fields appear to dominate higher order moments even at moderate nonlinearity leading to the reasonable qualitative behaviour of the model.

Supplementary data. Supplementary material and movies are available at https://doi.org/10.1017/jfm.2019...

Acknowledgements. The authors are grateful to an anonymous referee who pointed out the value of discussing viscous effects.

Funding. This work was funded by the UK Natural Environment Research Council under grant number NE/S009922/1. 
Declaration of Interests. The authors report no conflict of interest.

Author ORCID. E. Johnson, https://orcid.org/0000-0001-7129-8471; M. Crowe, https://orcid.org/0000-0002-9916-2653

\section{Appendix A. The inertial frame}

The analysis above considers the fluid velocity $\boldsymbol{u}$ relative to the static inertial $O x^{\prime} y z$ frame in terms of the coordinate $x$ moving with the dipole. It may be of value to briefly note how the results above conform to standard results in the inertial frame. For the two-dimensional slice model of $\S 2$ consider a region $R$ composed always of the same fluid elements bounded by the closed curve $C$ and sufficiently large to bound the region occupied by the LC-dipole throughout the motion. Then the transport theorem shows that

$$
\frac{\mathrm{d}}{\mathrm{d} t} \int_{R} \rho u \mathrm{~d} V=-\oint_{C} p \hat{\boldsymbol{x}} \cdot \hat{\boldsymbol{n}} \mathrm{d} s, \quad \frac{\mathrm{d}}{\mathrm{d} t} \int_{R} \frac{1}{2}\left(\rho \boldsymbol{u}^{2}+\rho b^{2}\right) \mathrm{d} V=-\oint_{C} p \boldsymbol{u} \cdot \hat{\boldsymbol{n}} \mathrm{d} s,
$$

where the decay of the dipole velocity field ensures that the leading order momentum and energy integrals are bounded, although the momentum integral depends on the shape of $C$ (Batchelor 1967). For sufficiently large domains the momentum and energy integrals can be written as

$$
\mu=\int_{R} \rho u \mathrm{~d} V=-\int z \eta \mathrm{d} V, \quad E=\int_{R} \frac{1}{2} \rho \boldsymbol{u}^{2} \mathrm{~d} V=-\frac{1}{2} \int \psi \eta \mathrm{d} V .
$$

The boundary integral terms omitted in deriving the energy integral in (A 2) vanish at large distance to give a unique definition. The momentum integral requires an interpretation in terms of the impulse required to set up the flow from rest (Lamb 1932; Batchelor 1967). Combining (A 1) and (A 2) gives the results used in the weak stratification limit here

$$
\frac{\mathrm{d} \mu}{\mathrm{d} t}=-\oint_{C} p \hat{\boldsymbol{x}} \cdot \hat{\boldsymbol{n}} \mathrm{d} s, \quad \frac{\mathrm{d} E}{\mathrm{~d} t}=-\oint_{C} p \boldsymbol{u} \cdot \hat{\boldsymbol{n}} \mathrm{d} s .
$$

Since the LC-dipole is an exact solution of the Euler equations it suffers no drag in inviscid flow and so the sole contribution to the drag force integrals in (A 3) comes from the internal wave drag which gives a contribution to the integrals of (A 3) that remains constant at large distances. The slower decay of the momentum boundary terms in (A 2) may lend support to the energy-work balance being more fundamental in $\S \S 3,4$.

\section{REFERENCES}

Batchelor, G. K. 1967 An Introduction to Fluid Dynamics. Cambridge: Cambridge University Press.

Bretherton, F. P. 1967 The time-dependent motion due to a cylinder moving in an unbounded rotating or stratified fluid. J. Fluid Mech. 28, 545-570.

Brion, V., SipP, D. \& JACQUin, L. 2014 Linear dynamics of the LambChaplygin dipole in the two-dimensional limit. Phys. Fluids 26, 064103, arXiv: https://doi.org/10.1063/1.4881375.

Burns, K. J., Vasil, G. M., Oishi, J. S., Leconnet, D. \& Brown, B. P. 2020 Dedalus: A flexible framework for numerical simulations with spectral methods. Phys. Rev. Res. 2, 023068.

Carr, M., Davies, P. \& Hoebers, R. 2015 Experiments on the structure and stability of 
mode-2 internal solitary-like waves propagating on an offset pycnocline. Phys Fluids 27, 046602.

Couder, Y. \& Basdevant, C. 1986 Experimental and numerical study of vortex couples in two-dimensional flows. J. Fluid Mech. 173, 225-251.

Crowe, M. N., Kemp, C. J. D. \& Johnson, E. R. 2021 The decay of Hill's vortex in a rotating flow (under review).

Delbende, I. \& Rossi, M. 2009 The dynamics of a viscous vortex dipole. Physics of Fluids 21, 073605.

Flierl, G. R. 1987 Isolated eddy models in geophysics. Annu. Rev. Fluid Mech. 19, 493-530, arXiv: https://doi.org/10.1146/annurev.fl.19.010187.002425.

Flierl, G. R. \& Haines, K. 1994 The decay of modons due to Rossby wave radiation. Phys. Fluids 6, 3487-3497, arXiv: https://doi.org/10.1063/1.868405.

Flierl, G. R., Stern, M. E. \& Whitehead, J. A. 1983 The physical significance of modons: Laboratory experiments and general integral constraints. Dyn. Atmos. Oceans 7, 233-263.

VAN DE Fliert, B. W. 1996 The viscous modulation of Lamb's dipole vortex. Physics of Fluids 8, 1975-1977, arXiv: https://doi.org/10.1063/1.868977.

Flór, J. B. \& van HeiJst, G. J. F. 1994 An experimental-study of dipolar vortex structures in a stratified fluid. J. Fluid Mech. 279, 101-133.

Flór, J. B., van Heijst, G. J. F. \& Delfos, R. 1995 Decay of dipolar vortex structures in a stratified fluid. Phys. Fluids 7, 374-383.

Ford, R., McIntyre, M. E. \& Norton, W. A. 2000 Balance and the slow quasimanifold: some explicit results. J Atmos Sci 57, 1236-1254.

van Geffen, J. H. G. M. \& van Heijst, G. J. F. 1998 Viscous evolution of 2D dipolar vortices. Fluid Dynamics Research 22, 191-213.

Gorodtsov, V.A. \& Teodorovich, E.V. 1983 Study of internal waves in the case of rapid horizontal motion of cylinders and spheres. Fluid Dyn. 17, 893-898.

Hill, M. J. M. 1894 On a spherical vortex. Phil. Trans. R. Soc. 185, 213-245.

Kamachi, M. \& Honji, H. 1982 Steady flow patterns of internal solitary bulges in a stratified fluid. Phys Fluids 25, 1119-1120.

LAMB, H. 1932 Hydrodynamics, 6th edn. CUP.

Larichev, V. D. \& Reznik, G. M. 1976 Two-dimensional solitary Rossby waves. Dokl. Akad. Nauk SSSR 231, 12-13.

Long, R.R. 1955 Some aspects of the flow of stratified fluids. III: Continuous density gradients. Tellus 7, 341-357.

McWilliams, J. C. \& Zabusky, N. J. 1982 Interactions of isolated vortices. 1. Modons colliding with modons. Geophys. Astrophys. Fluid Dyn. 19, 207-227.

Meleshio, V. V. \& van Heijst, G. J. F. 1994 On Chaplygin's investigations of twodimensional vortex structures in an inviscid fluid. J. Fluid Mech. 272, 157-182.

Nguyen Duc, J.-M. \& Sommeria, J. 1988 Experimental characterization of steady twodimensional vortex couples. J. Fluid Mech. 192, 175-192.

Nielsen, A. H. \& Rasmussen, J. J 1997 Formation and temporal evolution of the Lamb dipole. Phys. Fluids 9, 982-991, arXiv: https://doi.org/10.1063/1.869193.

NyCANDER, J. \& Isichenko, M. B. 1990 Motion of dipole vortices in a weakly inhomogeneous medium and related convective transport. Phys. Fluids 2, 2042-2047, arXiv: https://doi.org/10.1063/1.859425.

Salloum, M., Knio, O. M. \& Brandt, A. 2012 Numerical simulation of mass transport in internal solitary waves. Phys Fluids 24, 016602.

Velasco Fuentes, O. U. \& van Heijst, G. J. F. 1994 Experimental-study of dipolar vortices on a topographic $\beta$-plane. J. Fluid Mech. 259, 79-106. 